PHYSICAL REVIEW A 82, 030701(R) (2010)

\title{
Isotope effect in ion-atom collisions
}

\author{
P. Barragán \\ Instituto de Física Fundamental, CSIC, Serrano 123, Madrid E-28006, Spain \\ L. F. Errea, L. Méndez, and I. Rabadán* \\ Laboratorio Asociado al CIEMAT de Física Atómica y Molecular en Plasmas de Fusión, Departamento de Química, \\ Universidad Autónoma de Madrid, Madrid E-28049, Spain
}

(Received 7 May 2010; revised manuscript received 29 July 2010; published 28 September 2010)

\begin{abstract}
We explain the origin of the unusual large isotopic dependence found in charge-transfer cross sections for $\mathrm{H}(\mathrm{D}, \mathrm{T})^{+}+\mathrm{Be}$ collisions. We show that this large effect appears in a semiclassical treatment as a consequence of the mass dependence of the charge-transfer transition probabilities, which is due to the variation of the radial velocity in the region where the nonadiabatic transitions take place. The possibility of finding such a large isotope effect in other collision systems is discussed.
\end{abstract}

DOI: 10.1103/PhysRevA.82.030701

PACS number(s): 34.70.+e, 34.10.+x

In a previous work [1], we carried out an $a b$ initio quantal calculation of total charge-transfer (CT) cross sections, $\sigma^{\mathrm{H}}$, $\sigma^{\mathrm{D}}$, and $\sigma^{\mathrm{T}}$, for collisions of $\mathrm{H}^{+}, \mathrm{D}^{+}$, and $\mathrm{T}^{+}$with Be:

$$
\mathrm{H}^{+}\left(\mathrm{D}^{+}, \mathrm{T}^{+}\right)+\mathrm{Be}\left(1 s^{2} 2 s^{2}\right) \rightarrow \mathrm{H}(\mathrm{D}, \mathrm{T})(1 s)+\mathrm{Be}^{+}\left(1 s^{2} 2 p\right) .
$$

This collision system is very important in nuclear fusion since $\mathrm{Be}$ is proposed as a first-wall material of ITER [2]. In general, $\mathrm{CT}$ cross sections at low energies are required for modeling and diagnostics of plasmas. Furthermore, tritium inventory is a crucial problem in the development of the fusion reactor. A remarkable result of Ref. [1] is the very large isotope effect, with $\sigma^{\mathrm{H}}>10 \sigma^{\mathrm{D}}$ and $\sigma^{\mathrm{D}}>5 \sigma^{\mathrm{T}}$, at collision energies in the laboratory reference frame $E_{\text {lab }}<0.1 \mathrm{eV} / \mathrm{u}$. The main goal of the present work is to explain the origin of this strong isotopic dependence and to identify the factors that would lead to similar effects in other collision systems.

Isotope effects in ion-atom collisions were studied in Ref. [3], where a simple expression for the nuclear mass dependence of the cross section was derived, based on the use of the Landau-Zener model. It was also shown that the isotope effect is mainly a kinematic effect due to the mass dependence of the relative radial velocity. Merged-beam experiments have systematically looked for a large target isotope effect (see [4] and references therein) in several ion collisions with $\mathrm{H}$ and $\mathrm{D}$, but the results have shown a relatively small variation of the $\mathrm{CT}$ cross section on the nuclear reduced mass, $\mu$. As an example, the collisions of $\mathrm{N}^{2+}$ with $\mathrm{H}(1 s)$ and $\mathrm{D}(1 s)$ lead to very similar CT cross sections (see [5] and Fig. 4 below). A second example is $\mathrm{Si}^{4+}+\mathrm{H}(\mathrm{D})$ collisions, where the calculations of Pieksma et al. [6] pointed out a significant deviation from the simple Langevin model [7], with the CT cross section from $\mathrm{H}$ larger than that from $\mathrm{D}$ by a factor of 1.9 , at the lowest energy of their calculation $\left(E_{\text {lab }}=0.02 \mathrm{eV} / \mathrm{u}\right)$. This result has been confirmed by the experiment of Ref. [4]. However, this dependency is not as large as that found in Ref. [1]. On the other hand, a noticeable isotope effect at relatively

*ismanuel.rabadan@uam.es high collision energies $\left(30 \mathrm{eV} / \mathrm{u}<E_{\text {lab }}<500 \mathrm{eV} / \mathrm{u}\right)$ is been pointed out in Ref. [8] for CT in $\mathrm{He}^{2+}+\mathrm{H}(1 s)$ collisions, where rotational transitions are very important. The effect observed in this work can also be interpreted as a kinematic effect, where the total cross section changes with the reduced mass because the distance of closest approach increases when the reduced mass decreases. For some nuclear trajectories, the distance of closest approach becomes larger than the internuclear distances where the nonadiabatic transitions take place, which leads to smaller cross sections for the lighter isotopic species.

In our calculations the collision wave function is a solution of the nonrelativistic Schrödinger equation, and it does not include spontaneous emission; therefore, radiative charge transfer is not considered. The collision wave function of Ref. [1] was expanded in terms of a large molecular basis that included a common reaction coordinate [9] to correctly fulfill the boundary conditions. This reaction coordinate modifies the nonadiabatic couplings by adding mass-dependent terms to the nuclear gradient integrals. Although in general these terms are required to remove spurious isotopic dependencies, we have checked that in this particular case they do not significantly modify the actual CT total cross sections, and therefore they are not considered in the following discussion.

The total cross section for reaction (1) [see Fig. 1(a)] shares some characteristics with other collision systems; that is, the Langevin-like behavior at low velocities, with cross sections proportional to $v^{-1}$ ( $v$ is the relative velocity), and the presence of resonant structures, which have been found in calculations for other collisions (see Refs. [10,11] and references therein). In addition, at low energies $\left(v \approx 10^{-3}\right.$ a.u., which corresponds to $E_{\text {lab }} \approx 25 \mathrm{meV} / \mathrm{u}$ ) the cross section ratios are $\sigma^{\mathrm{H}} / \sigma^{\mathrm{D}} \approx 20$ and $\sigma^{\mathrm{H}} / \sigma^{\mathrm{T}} \approx 200$, which are very large values not found previously. In order to analyze the origin of this isotope effect and, in particular, to find out whether quantal effects are involved, it is useful to introduce two-state, quantal, and semiclassical simplified models, which will also allow us to generalize our findings.

At low-impact energies, the CT process (1) takes place through transitions between two molecular states [those dissociating into $\mathrm{H}^{+}+\mathrm{Be}\left(1 s^{2} 2 s^{21} \mathrm{~S}\right)$ and $\mathrm{Be}^{+}\left(1 s^{2} 2 p^{2} \mathrm{P}\right)+$ $\mathrm{H}(1 s)$ ], so we have constructed a two-state model in terms 


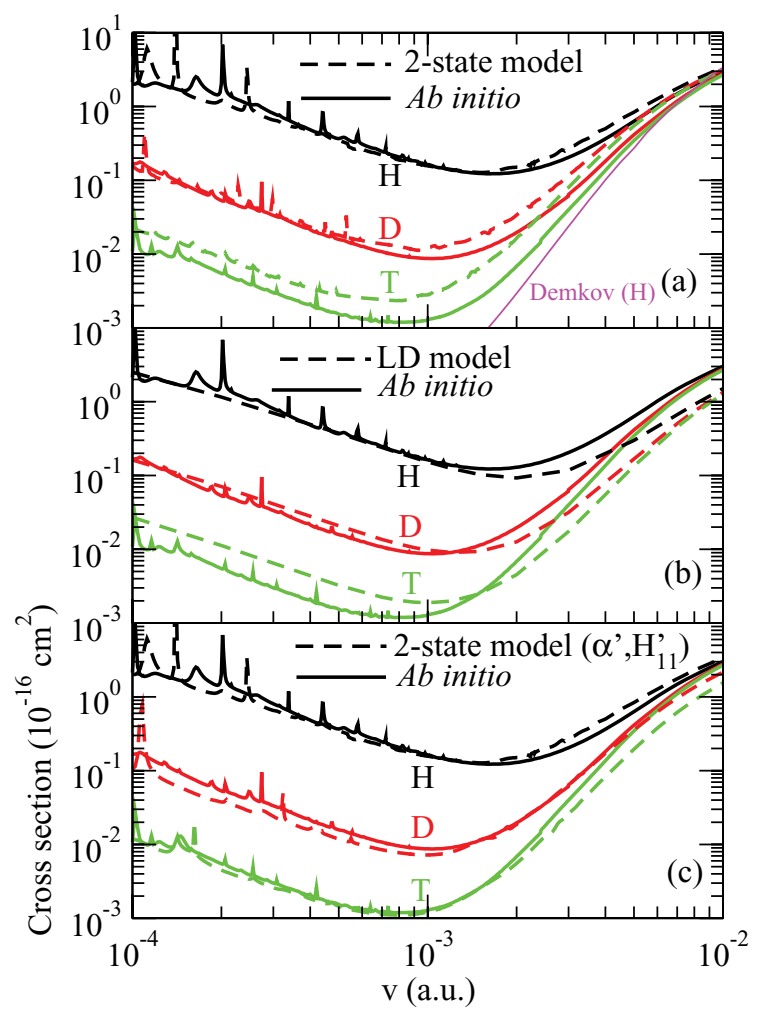

FIG. 1. (Color online) CT total cross sections for $\mathrm{H}(\mathrm{D}, \mathrm{T})^{+}+$ $\mathrm{Be}$, collisions. The ab initio results are compared to different twostate models: (a) The model of Eq. (2). (b) The Langevin-Demkov (LD) model. (c) The model of Eq. (2) where we have changed the polarizability by scaling it with the reduced mass and the asymptotic value of $H_{22}-H_{11}$ in order to get a mass-independent value of the diabatic energy difference at $R=R_{p}$.

of two diabatic states, similar to that of Refs. [12,13]. Explicitly:

$$
\begin{gathered}
H_{22}-H_{11}=0.0102+72.6 \exp (-1.35 R)-\frac{36.144}{2 R^{4}}, \\
H_{12}=0.804 \exp (-0.820 R) .
\end{gathered}
$$

The cross sections obtained by numerically integrating the ensuing system of differential equations are plotted in Fig. 1(a), where one can note that this model reproduces the main features of the $a b$ initio calculation.

A more simple model is obtained by assuming a constant energy gap (Demkov model) [14,15]:

$$
\begin{gathered}
H_{22}-H_{11}=\Delta H, \\
H_{12}=A \exp (-\lambda R) .
\end{gathered}
$$

In the present case, by fitting the ab initio energy curves near the transition region, we obtain $\Delta H=0.0066$ Hartree, with $A=0.804$ Hartree and $\lambda=0.82$ bohr $^{-1}$ as in Eq. (2).

Although the model is useful at relatively high velocities, the lack of the ion-induced dipole interaction leads to a cross section that decreases at low energies [see Fig. 1(a)], not showing the Langevin-type behavior.

In order to extend the Demkov method to low energies and to check if the isotope effect can be reproduced by a semiclassical treatment, we applied the Langevin-Demkov
(LD) model; this is similar to the Langevin-Landau-Zener model suggested in Ref. [16] and previously applied for several ion-H $(1 s)$ collisions in Ref. [17]. The assumptions of the model are as follows:

1. The classical nuclear motion takes place in the adiabatic potential of the collision entrance channel; it is assumed that this potential has the simple ion-induced dipole form:

$$
V(R)=-\frac{q^{2} \alpha}{2 R^{4}},
$$

where $\alpha$ is the target polarizability and $q$ the projectile charge.

2. As in the Langevin model, the transitions leading to the CT reaction take place at impact parameters lower than $b_{\max }$, the maximum value that allows the surmounting of the centrifugal barrier [18]:

$$
b_{\max }=\left(\frac{2 q^{2} \alpha}{\epsilon}\right)^{1 / 4}
$$

where $\epsilon$ is the impact energy in the center-of-mass reference frame. The total cross section is therefore

$$
\sigma=2 \pi \int_{0}^{b_{\max }} b P(b) d b .
$$

In this expression we assume that the nonadiabatic transitions take place at internuclear distances smaller than $b_{\max }$, which is the most frequent situation.

3. The transition probability, $P$, is obtained by solving the system of differential equations derived from the two-state Hamiltonian matrix, Eq. (3), which leads to the analytical expression (see $[15,19])$

$$
P=\operatorname{sech}^{2}\left(\frac{\pi \Delta H}{2 \lambda v_{r}}\right) \times \text { oscillatory terms. }
$$

$v_{r}$ is the radial velocity,

$$
v_{r}=v\left[1-\frac{2 V\left(R_{p}\right)}{\mu v^{2}}-\frac{b^{2}}{R_{p}^{2}}\right]^{1 / 2},
$$

which is evaluated at $R=R_{p}$, obtained from the relation

$$
\Delta E\left(R_{p}\right)=\sqrt{2} \Delta H,
$$

with $\Delta E(R)$ being the energy difference between the adiabatic states. In the present case, the parameters of the two-state model of Eq. (3) lead to $R_{p}=6.68$ a.u.

The distance $R_{p}$ indicates the region of internuclear separations where transitions take place. We have checked that for the impact energies considered in this work, $b_{\max }>R_{p}$, and the transitions take place at distances smaller than the position of the centrifugal maximum, in accordance with the assumption of Eq. (6). It can be noted that, for constant $v$, the cross section depends on $\mu$ through Eqs. (5) and (8). The increase of $\mu$ leads to a decrease of the acceleration that depends on the ratio $\alpha / \mu$. As shown in Ref. [20], the inclusion of plane-wave translation factors in the Demkov model modifies the nonoscillatory part of the transition probability (7) by substituting $\Delta H$ by $\Delta H+v^{2} / 2$, which is not significant at the collision energies considered in this work.

We have applied the LD model to $\mathrm{H}^{+}+\mathrm{Be}$ collisions and the results are shown in Fig. 1(b). Although there are some differences between the model and the numerical cross section for the collision with tritium, and the obvious fact that 


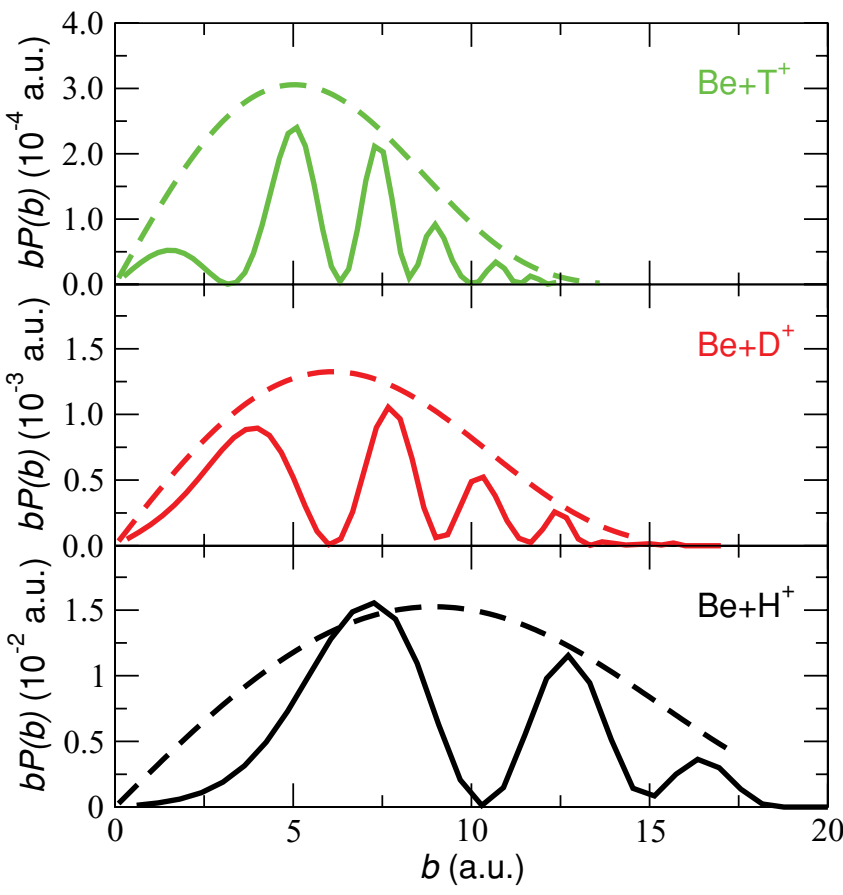

FIG. 2. (Color online) Product of the impact parameter $b$ and charge-transfer transition probabilities $P(b)$ obtained in the ab initio 28-state calculation (solid lines) of Ref. [1], compared to the nonoscillatory part of the Demkov expression (7) (dashed lines) for collisions $\mathrm{Be}+\mathrm{H}(\mathrm{D}, \mathrm{T})^{+}$, as indicated in the figure, and for $v=0.001 \mathrm{a} . \mathrm{u}$.

the semiclassical model is unable to reproduce the resonant structures, the model shows a satisfactory agreement with the numerical calculation, and we can conclude that the isotopic dependence is a consequence of trajectory effects. Moreover, the agreement supports the explanation that the huge isotope effect is not related to reaction coordinates (or the equivalent translation factors in the semiclassical formalism) corrections, which are included in the numerical treatment, but not in the LD model. A more stringent test of the model is provided by the transition probabilities plotted in Fig. 2, where the nonoscillatory part of the Demkov transition probabilities for $b<b_{\max }$ are compared with the ab initio ones.

An additional test on the explanation of the effect is shown in Fig. 1(c), where we have plotted the CT cross sections evaluated by integrating the system of differential equations from the model of Eq. (2) by changing the target polarizability instead of varying the nuclear reduced mass. In particular, we have considered three different values of the polarizability $\alpha^{\prime}=\frac{\mu_{\mathrm{BeH}}}{\mu} \alpha$ and a single reduced mass $\left(\mu=\mu_{\mathrm{BeH}}\right)$. The results show that the total cross section depends on $\mu$ through the fraction $\alpha / \mu$ [see Eq. (8)]. In this test calculation, we have also slightly modified the asymptotic energy gap, $H_{22}-H_{11}$, in order to get similar energy differences for the three values of $\alpha^{\prime}$ at the point $R=R_{p}$ [see Eq. (9)].

In order to gauge the potential relevance of the isotopic effect in plasma modeling, we have evaluated the rate coefficients $K_{p}(T)$ for the CT reactions (1) using

$$
K_{p}(T)=4 \pi\left(\frac{\mu_{p}}{2 \pi k_{B} T}\right)^{3 / 2} \int_{0}^{\infty} d v v^{3} \sigma_{p}(v) \exp \left(-\frac{\mu_{p} v^{2}}{2 k_{B} T}\right),
$$

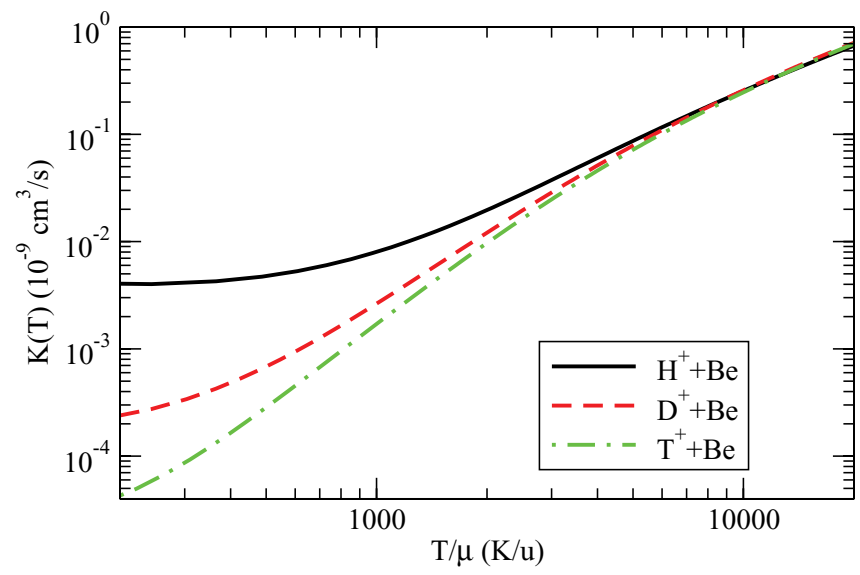

FIG. 3. (Color online) Rate coefficients for the CT reactions (1) as functions of the scaled temperature $T / \mu$. For the three isotopic variants, as indicated in the figure.

where $T$ is the temperature, $k_{B}$ is the Boltzman constant, and the subscript $p$ indicates the hydrogen isotope. The calculated values are shown in Fig. 3 as functions of the scaled temperature, $T / \mu_{p}$, to illustrate the part of the isotopic dependence of the rate coefficients that comes from the isotopic dependence of the $\mathrm{CT}$ cross section. These results indicate that a noticeable effect is expected at $T \approx 1000 \mathrm{~K}$ and, since the Be melting point is about $1560 \mathrm{~K}$, it should be relevant in modeling the plasma behavior near the first wall of tokamaks.

With respect to the comparison with other systems, we present in Fig. 4 the variation of the $\mathrm{CT}$ cross sections with the reduced mass for several ion-atom collisions, for a relative velocity of 0.001 a.u. (an impact energy of about $25 \mathrm{meV} / \mathrm{u}$ ). The results for $\mathrm{N}^{3+}+\mathrm{H}$ shown in this figure were obtained by integrating the model of Ref. [12]. Cross sections for $\mathrm{N}^{2+}+\mathrm{H}$ were obtained ab initio using the molecular basis set of Ref. [5], and those for $\mathrm{H}^{+}+\mathrm{Be}$ are also ab initio data. Our results point out that the magnitude of the isotope effect depends on the interaction potential between the colliding particles (note that $\alpha_{\mathrm{H}}=4.50$ a.u., $\alpha_{\mathrm{Be}}=36.14$ a.u.). Nevertheless, the isotope effect is not completely explained by the trajectory effect

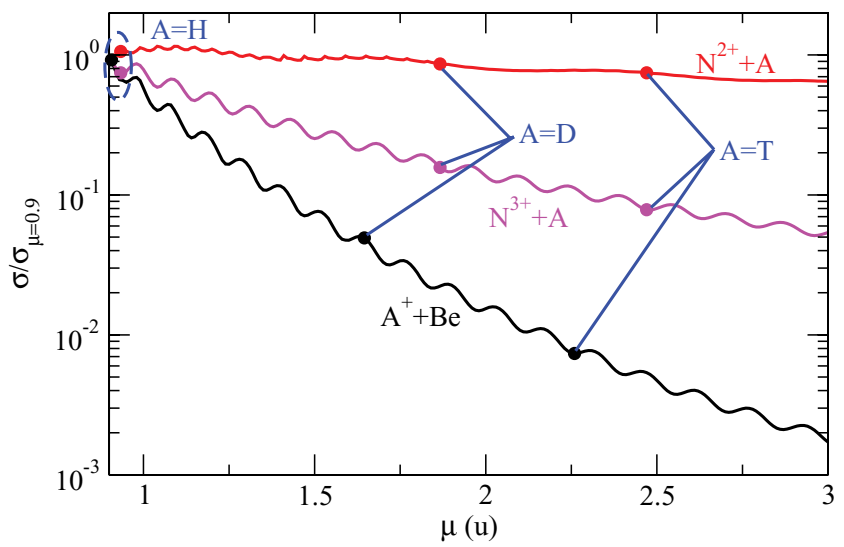

FIG. 4. (Color online) Comparison of the mass dependence of the CT total cross sections for $\mathrm{N}^{2+}+A, \mathrm{~N}^{3+}+A$, and $A^{+}+\mathrm{Be}$ collisions, where $A$ indicates a fictitious hydrogen isotope with variable mass, at a relative velocity of $v=0.001$ a.u. $\left(E_{\mathrm{lab}} \approx\right.$ $25 \mathrm{meV} / \mathrm{u}$ ). 
in the region where transitions take place. In particular, the isotopic dependence of $\mathrm{CT}$ cross section in $\mathrm{N}^{3+}+\mathrm{H}$ collisions is markedly smaller than the corresponding one for $\mathrm{H}^{+}+\mathrm{Be}$, although the products $q^{2} \alpha$ are similar for both collisions. The main difference between these two CT reactions is that the former one takes place through transitions in a sharp avoided crossing, well described by means of the Landau-Zener model, while in the latter process the diabatic energy curves are almost parallel, which is well described by means of the Demkov model. A similar argument could explain that the isotope effect in the $\mathrm{Si}^{4+}+\mathrm{HCT}$ process [4] is smaller than that in $\mathrm{H}^{+}+\mathrm{Be}$.

A different behavior is found for other ion-H collisions (e.g., $\mathrm{He}^{2+}+\mathrm{H}$ [8], $\mathrm{Li}^{+}+\mathrm{H}$ [21]), where the total CT cross section increases with $\mu$. In these collisions the nonadiabatic transitions take place at relatively short internuclear distances where the interaction potential is repulsive, which leads to a reduction of the range of $b$ in the integral (6) as $\mu$ decreases, with a relatively small isotopic dependency of the transition probability. In this respect, it must be noted that in these two examples the CT reaction is endothermic and the exit channel becomes closed at low energies, yielding a threshold in the total cross section as function of the projectile velocity, while the examples of Fig. 4 are exothermic reactions with large cross sections at low energies. The CT process,

$$
\mathrm{H}(\mathrm{D}, \mathrm{T})(1 s)+\mathrm{Be}^{+}\left(1 s^{2} 2 p^{2} \mathrm{P}\right) \rightarrow \mathrm{Be}\left(1 s^{2} 2 s^{2}\right)+\mathrm{H}(\mathrm{D}, \mathrm{T})^{+},
$$

which is the inverse of reaction (1), is an additional example of the former situation. The numerical calculation with the 28-molecular-state basis of Ref. [1] leads to the total cross section plotted in Fig. 5. These results are practically identical to those obtained from the two-state model of Eq. (2), where, using microreversibility, the total cross section for reaction (11) is evaluated from the $S$-matrix elements of reaction (1):

$$
\sigma_{j i}\left(k_{j}\right)=\frac{k_{i}^{2}}{k_{j}^{2}} \sigma_{i j}\left(k_{i}\right),
$$

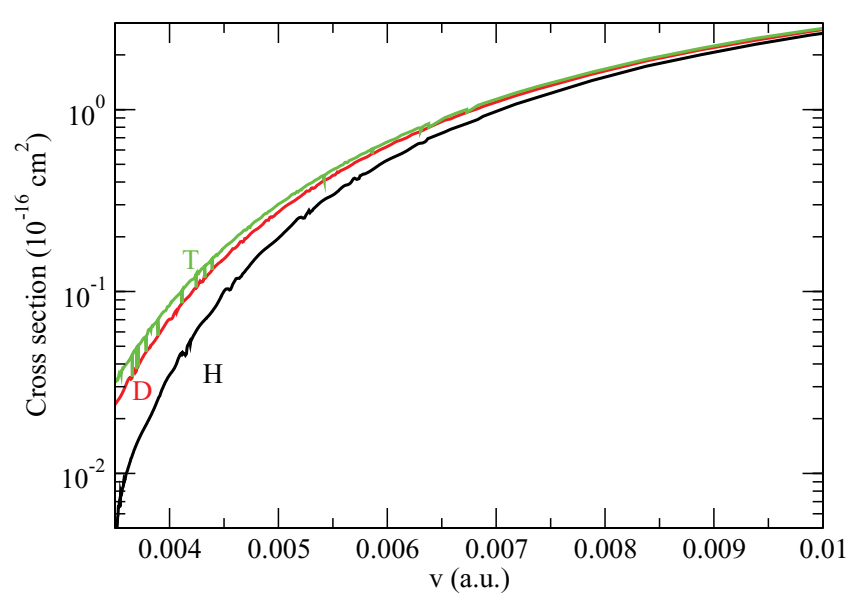

FIG. 5. (Color online) Total cross section for the CT reaction (11) as a function of the relative velocity.

where $k_{i}=\sqrt{2 \mu\left[E-E_{i}(\infty)\right]}$ and $k_{j}=\sqrt{2 \mu\left[E-E_{j}(\infty)\right]}$. $E_{i, j}(\infty)$ are the energies of the entrance channels of reactions (1) and (11), respectively, with $E_{i}(\infty)-E_{j}(\infty)=0.0102$ Hartree $=0.277 \mathrm{eV}$.

In summary, we have shown that the very large isotope effect exhibited by the total CT charge transfer in $\mathrm{H}^{+}+\mathrm{Be}$ collisions is a consequence of the dependence of the transition probabilities on the radial velocity [Eq. (8)], which notably changes with the nuclear reduced mass because of the large polarizability of the Be atom. Strong isotope effects are therefore expected for ion collisions with atoms or molecules with large polarizabilities when the ion-induced dipole interaction is the dominant contribution to the ion-target interaction at the internuclear distances where the $\mathrm{CT}$ process takes place.

This work has been supported by Project ENE2007-62934 of the Ministerio de Ciencia e Innovación (Spain).
[1] P. Barragán, L. F. Errea, L. Méndez, I. Rabadán, and A. Riera, J. Phys. B 41, 225202 (2008).

[2] ITER Physics Basis, Nucl. Fusion 39, 2391 (1999).

[3] P. C. Stancil and B. Zygelman, Phys. Rev. Lett. 75, 1495 (1995).

[4] C. C. Havener, D. G. Seely, J. D. Thomas, and T. J. Kvale, AIP Conf. Proc. 1099, 150 (2009).

[5] P. Barragán, L. F. Errea, L. Méndez, I. Rabadán, and A. Riera, Phys. Rev. A 74, 024701 (2006).

[6] M. Pieksma, M. Gargaud, R. McCarroll, and C. C. Havener, Phys. Rev. A 54, 13(R) (1996).

[7] P. Langevin, Ann. Chim. Phys. 5, 245 (1905).

[8] N. Stolterfoht, R. Cabrera-Trujillo, Y. Öhrn, E. Deumens, R. Hoekstra, and J. R. Sabin, Phys. Rev. Lett. 99, 103201 (2007).

[9] W. R. Thorson and J. B. Delos, Phys. Rev. A 18, 135 (1978).

[10] P. Barragán, L. F. Errea, F. Guzmán, L. Méndez, I. Rabadán, and I. Ben Itzhak, Phys. Rev. A 81, 062712 (2010).

[11] A. K. Belyaev, P. S. Barklem, A. S. Dickinson, and F. X. Gadéa, Phys. Rev. A 81, 032706 (2010).
[12] M. Rittby, N. Elander, E. Brändas, and A. Bárány, J. Phys. B 17, L677 (1984).

[13] M. Roudjane, R. McCarroll, and D. Rabli, J. Phys. B 40, 2491 (2007).

[14] W. E. Meyerhof, Phys. Rev. Lett. 31, 1341 (1973).

[15] Y. N. Demkov, Sov. Phys. JETP 18, 138 (1964).

[16] A. M. Chang and D. E. Pritchard, J. Chem. Phys. 70, 4524 (1979).

[17] P. Barragán, L. F. Errea, F. Guzmán, L. Méndez, I. Rabadán, and A. Riera, J. Phys. Conf. Ser. 58, 227 (2007).

[18] G. Gioumousis and D. P. Stevenson, J. Chem. Phys. 29, 294 (1958).

[19] E. E. Nikitin and S. Y. Umanskii, Theory of Slow Atomic Collisions (Springer-Verlag, Berlin, 1984).

[20] S. J. Pfeifer and J. D. Garcia, Phys. Rev. A 23, 2267 (1981).

[21] L. F. Errea, F. Guzmán, L. Méndez, B. Pons, and A. Riera, Phys. Rev. A 77, 012706 (2008). 